25. Rogers, W. K. - Trans. Amer. Ophthal. Soc., Vol. IX, pp. 525-529, 1900-02.

26. Scherl.-Arch. f. Augenheilk., Bd. XXVIII, S. 26, 1893.

27. Schweinitz, G. E. de-Therap. Gazette, Detroit, Series 3, Vol. IX, pp. 11-18, 1893.

28. Sinclair, A. H. H.-Jl. of Path. and Bact., Edinburgh and London. Vol. VII, pp. 295-307, 1900-01.

29. Snell, S.-Trans. Ophthal. Soc. U.K., Vol. XVI, p. 72, 1895-6.

30. Sourdville, G.-Arch. d'Ophtal., Nantes, July, 1923. Brit. Jl. of Ophthal., Vol. VIII, p. 594, 1924.

31. Stillson, J. O.-Trans. West. Ophthal. Otol., etc., Assoc. Ophthal. Div., St. Louis, pp. 28-39, 1898.

Amer. Jl. of Ophthal., Vol. XV, pp. 129-141.

32. Sutphen, T. Y.-Trans. Amer. Ophthal. Soc., Vol. VI, pp. 306-308, $1891-3$.

33. Webster.-New York Med. Jl., Vol. LIII, pp. 326-330, 1891

34. Weiner, M.-Arch. of Ophthal., Vol LIII, pp. 368-380, 1924.

35. Wray, C. - Trans. Ophthal. Soc. U.K., Vol. XVI, p. 63, 1895-6.

\title{
DEFECTIVE SIGHT AND SIGHT EDUCATION
}

BY

Kenneth R. Smith, M.D.(Lond.)

KEW

Strabismus is described in a very well known text book on diseases of the eye under a section entitled "Disorders of Motility of the Eye." The word itself is defined as " a general term applied to all those conditions in which the visual axes assume a position relative to each other different from that required by the physiological conditions." When both eyes are directed to the same object, and are seen to follow it when it moves, it is certain that both eyes see it, and that they are in their true physiological position. Thus the physiological conditions of normal human sight are brought about when hoth eyes fix the same object. The condition of sight in concomitant strabismus is that only one eye fixes the object looked at, and the other does not fix it. The disorder of motility is, therefore, the failure of a movement which should occur under normal conditions, that is, the movement of fixation fails in one eye or in the other.

Further, although the power to see with each eye may exist, and in many cases of concomitant strabismus the sight with each eye separately is good, the power to see with both eyes at the same time is absent. This is a matter for observation, not for discussion. Let any child with concomitant strabismus look at an object, a pencil, for example, and ask him how many pencils he sees. His answer is " one." The eye which sees the pencil, fixes it. The eye which does not fix it, does not see it. for there is no double vision, 
and the conditions which make single vision possible with both eyes do not exist.

The observations are carried a step farther by covering the eye which sees the pencil. The squinting eye in most cases is then seen to fix the object, and the patient sees one pencil as before. The conditions of sight are thus the same when the sound eye is prevented from seeing as when it sees; in either case only one eye sees the pencil, the other eye deviates from its physiological position.

The observations may be completed by means of the binoscope. This apparatus enables most children with concomitant strabismus to see simultaneously with both eyes, and nearly all of them, not only see the object looked at with both eyes, but fix it with both eyes simultaneously, so that binocular vision is brought about, and all signs of strabismus vanish for the time. In some there is a momentary "double vision," which serves to demonstrate the sequence of events, namely, that simultaneous vision of the object with both eyes precedes the fixation of it by both eyes, and in some the diplopia is of longer duration. Now remove the binoscope; simultaneous vision with both eyes ceases, and the deviation, the disorder of motility, is observed as at first.

It is thus clear that in these cases the visual axes assume the position relative to one another required by the physiological conditions, when both eyes are enabled to see simultaneously the same object, and that they cease to maintain the position when the power to see it simultaneously ceases.

When the movement of a particular switch serves to switch on an electric lamp, and again to switch it off, it is certain that the movement of the switch controls the current in the lamp. In these cases of concomitant strabismus simultaneous vision of the object by both eyes causes all signs of the disorder to vanish, and the loss of the simultaneous vision is followed by their reappearance, and there is the same certainty that simultaneous vision of the same object by both eyes controls the disorder. Moreover there is no evidence of any motor defect. The movements of fixation are absent when the afferent stimulus which brings them about is absent, but take place in their natural perfection when the stimulus is present. 'The disorder is a sensory one.' It is a defect of vision, but not of the eyes or of the parts which move them, for each eye may, and often does, perform its normal functions separately from the other. The defect is in the visual centres. Retinal images of an object do not constitute the sensation of it. They may lead to sensation or they may not. We have no proof of subjectivity or modifications of consciousness apart from the action of the cerebral hemispheres. Retinal impressions must reach and induce molecular changes in the cells of the visual centres to excite a visual 
sensation, which is the subjective side of activity in these cells. Moreover we do not fix every object in the field of vision. When an object attracts mental attention, then fixation by the eye which sees it follows. When therefore an eye fixes an object, it is certain, not only that the object is seen, but that the sensation of it is associated with the concentration of consciousness which is meant by the word attention, that the sensation of it predominates over those of other objects included in the field of vision. The physical conception of the condition is intensified activity in those cortical cells which are concerned in the sensation of the particular object, with a diminution or, possibly, complete inhibition of activity in others.

In classification concomitant strabismus finds its true place among disorders of the brain, and in that division of them which relates to sensation.

The true sequence of events may be demonstrated by any one who desires to ascertain it. Thousands of operations for squint have failed to restore the physiological conditions of human sight. This almost universal failure shows the utter futility of treatment which ignores their true sequence. The movement of the eye to fix the object seen is a natural response to the stimulus which induces the sensation of it in the eye which sees it, and can be obtained in no other way than by bringing about the sensation. Sensation with both eyes simultaneously similarly brings about fixation by both eyes simultaneously, each by its own sensation.

Fixation of an object by the eye which sees it is an activity of great interest. It is automatic and is observed very early in life. A very young infant will fix a bright object and follow its movements with one eye, as $\mathrm{Mr}$. Worth has observed and recorded, and yet the movement of fixation presents typically the characteristics of true volitional action. As such one might describe it: the sensation of the object attracts attention, and awakens the desire to see it with the clearest vision, and the eye is moved so that the retinal image of the object falls on the punctum centrale to attain this clearest vision of it.

The following extract is explanatory of fixation :

"In the lower animals the control and co-ordination of movements are almost complete at birth, or require little education as compared with the prolonged helplessness of the human infant.

"Some birds start from the egg already fully equipped, like Athene from the head of Zeus. They are in a great measure mere ' conscious automata.' They are capable of acquiring sensory experience and association of ideas, but of little further motor acquisition beyond that with which they start in life. Their cortical motor centres count for little, and may be removed without causing much disturbance of their ordinary modes of activity. Rabbits 
require but comparatively short education to perfect their powers ; cats and dogs longer; but cats and dogs are already advanced in life, and have assumed the cares of paternity, or rather maternity, when the human infant can scarcely lift a finger in its own behalf.

"In proportion as volition predominates over conscious automatism, is education necessary to perfect the powers of movement; in the same proportion are the cortical centres developed; and in that proportion are the powers of movement paralysed by destruction of the motor centres of the hemispheres.

"In man volition is predominant; education is long and laborious; the faculty of special motor acquisition is unlimited; the cortical motor centres reach their highest development; and their removal causes such complete and enduring motor paralysis as to indicate that automatism in and by itself is scarcely detachable from the centres of consciousness and volition." (Ferrier: The Functions of the Brain.)

In the movement of the eye which sees an object to fix it automatism predominates. It is as automatic as those of birds described as conscious automatism, and is a completely organised co-ordinate motor acquisition at birth or very soon after; and the impulse for the attainment of clearest vision, which governs it, is instinctive. Walking has to be learned, speech gradually develops in early education, but fixation of an object by the eye which brings the sensation of it is an acquisition of the more elemental type ready for use without education. Phonation is similarly elemental ; an infant can cry; but the cultivation of this faculty in association with articulation, and the cultivation of fixation with both eyes simultaneously in association with nearly all our activities need education.

We thus begin life with each eye a separate alternative doorway to our clearest sense of sight. The sensory channel of each is complete, for the one co-ordinated movement which is needed for each eye to respond to the sight of an object by giving the clearest vision of it, is a completely organised sensori-motor acquisition. That is, fixation with each eye in response to its own sensation is as automatic as the movements of young ducks in water, or of young swallows in flight.

From this infantile vision the higher type of adult sight, binocular vision, develops by education. The process of education needs more investigation, but a few facts which bear on the subject may be mentioned.

(1) The field of vision with both eyes is wider than that of either eye alone. Any group of objects which extends completely across the wider field of simultaneous vision with both eyes, cannot be kept in view by one eye only; looking at such a group tends to induce activity in both eyes at the same time. This is the principle 
of the binoscope, which demonstrates the truth. of the statement. Further, if any object in this group attracts mental attention and is " fixed," it is as a rule fixed by both eyes. Such conditions must present themselves to the growing infant during its hours of wakefulness, and almost certainly begin the natural education of binocular vision.

(2) The acquisition of early purposive co-ordinate movements appears to be closely associated with the development of binocular vision. It is the natural sensory component in nearly all of them, and is the guiding sense of most skilled movements throughout life. The necessary attention is readily concentrated and retained in the process of acquiring co-ordinate movements, and the sense itself is educated in their acquisition. Pears' well known advertisement picture, " He wont be happy till he gets it," is a good illustration of the natural education of binocular vision; the child is training his movements and his sight in the effort to attain the object of his young desires.

(3) The natural education in maintaining binocular vision for near objects appears to belong to a later period of growth. The schoolboy may afford illustrations of it. He may take a clock to pieces to see how it works; or he may collect birds' eggs, butterflies or postage stamps; and the desire to distinguish one from the other retains his attention in examining details, and thus tends to maintain fixation with both eyes indefinitely without conscious effort. This natural tendency to observe and investigate thus apparently initiates another stage in the education of sight, that is, in establishing the physiological conditions of adult vision to replace completely those of infancy in prolonged near vision.

Civilised conditions impose upon children the prolonged use of the eyes in near vision in learning to read and to write before the power to retain binocular vision for near objects has been fully acquired, at a period when instability of the adult type of sight is marked by the liability to strabismus, and no attempt is made to assist the development of the sight to its adult type by education.

(4) The close association of sight with our intellectual life must be mentioned. "We cannot voluntarily concentrate attention on any idea which we cannot represent visually, either in its own characters, source or relations." If intellectual attention is mainly ideal vision, and the paths of activity in the re-presentation of ideation are the same as in the original presentation, that is, in observation, a point upon which physiological teaching-and that of Bain and Spencer, on other grounds, are agreed, the accuracy and facility of the re-presentation depend upon the natural use of the eyes in observation, that is, in establishing the coherent association of the sensori-motor acquisition. 
Strabismus thus marks a failure in the education of sight. The author above referred to defines heterophoria as latent strabismus. In concomitant strabismus when one eye fixes an object, the other eye does not fix it; this is the constant condition of sight; the failure to educate the sight of infancy to that of adult vision is complete, sight retains its infantile condition. In heterophoria the infantile condition of sight is not a constant one, but the liability that it may occur exists ; it may recur and persist just when simultaneous vision with both eyes is most needed.

The failure to educate sight to its normal condition of perfectly spontaneous binocular vision constitutes by far the most common defect of vision. It appears to leave the individual very often less efficient in respect of sight than the complete absence of binocular vision, which is the condition of sight in permanent squint. For in the complete absence of binocular vision there is no effort to maintain it, and there are no symptoms of asthenopia. The characteristic symptom of asthenopia is the constant effort to maintain clearest vision. Effort means fatigue when it is continuous ; hence the inability for prolonged close work. Repeated fatigue may lead to a condition of exhaustion and to neurasthenic conditions, as well as to indications of irritation in and about the eves themselves, which may be completely incapacitating. The condition of sight in concomitant strabismus thus appears in this respect to be a conservative one as compared with the vastly more common condition of incomplete development of normal adult sight.

Moreover, a condition which is capable of bringing about such severe irritative effects appears to be a very unfavourable one for the natural growth and development of the eyes themselves, and is likely to be a powerful factor in the enormous increase in defective vision, which is mainly defective growth and development. The natural environment, whose tendency is to correct in the process of growth what is defective, and to bring the ultimate result nearer to perfection, is in continued natural use.

There is ample evidence that either squint or heterophoria in the parents increases the liability to these defects in the children and that therefore the rapidity with which these defects spread tends ever to increase. The practical consideration of the subject is not therefore one for procrastination.

The facts are simple. Our sense of sight, like most human faculties, needs education, natural or otherwise, for its development to normal adult condition. Binocular vision, as a completely spontaneous function is the object sought by education. This is the most perfect sight and at the same time the most restful. Look through a stereoscope at any good stereoscopic picture. There is no sense of effort; observation becomes a pleasure in the perfection 
of the sense, and this is the environment for true evolution. " $\mathrm{Na}$ profit grows where is no pleasure ta'en," says Shakespeare of education in general. Nearly all our activities depend upon sight, and the adult sight with its marvellous appreciation of space is the only condition of the sense which leads to greatest perfection of the movements in space which depend upon it, that is, in most movements of skill; and its perfect restfulness alone affords the condition best adapted for use in observation and in prolonged near eye-work.

There is abundant evidence that natural education of sight under civilised conditions needs rational help. 'The words of Ferrier, above quoted, describe the physiological basis of education, by which the human being has a share in his own creation or evolution, less maybe than we think, but enough to make very great the responsibility for failure to provide education when it is obviously needed, and when its attainment is simple.

The facts point clearly to the failure in the power to retain simultaneous vision with both eyes for near eye-work in the early years of life, and as clearly show the necessity for training to attain stability in simultaneous vision with both eyes during these early years. Provided that the natural use of both eyes is thus assured, it appears probable that the ordinary use of them will complete the education.

\title{
NOTE ON TRAUMATIC ASPHYXIA WITH OCULAR COMPLICATIONS
}

\author{
BY \\ J. Bruce Hamilton \\ SENIOR HOUSE SURGEON, ROYAL LONDON OPHTHALMIC HOSPITAL
}

R. W. R., aged 15 years, was leaving his work at 6 p.m. on September 2, 1929, when he was caught between the door and floor of a lift and his chest was severely compressed for a period of not more than 60 seconds : on this point he is very clear. He was immediately taken to hospital where he was detained two hours and then sent home. On arriving at his home at 8.45 p.m. his mother noticed that his face was swollen and that his eyes were blood-shot, and he was put immediately to bed. Next day there was marked swelling of the neck and face, both eyes were red and the patient was drowsy and could remember nothing of the accident. His eyesight did not appear to be affected. At the end of 7 days his memory had returned : at the end of 9 days all swelling had dis- 